\title{
BLOWUP OF SOLUTIONS OF THE HYDROSTATIC EULER EQUATIONS
}

\author{
TAK KWONG WONG
}

(Communicated by Walter Craig)

\begin{abstract}
In this paper we prove that for a certain class of initial data, smooth solutions of the hydrostatic Euler equations blow up in finite time.
\end{abstract}

\section{INTRODUCTION}

We consider the hydrostatic Euler equations 1 in a two-dimensional tube $\mathbb{R} \times$ $[0,1]=\{(x, y) ;-\infty<x<\infty, 0 \leq y \leq 1\}$ :

$$
\begin{aligned}
\partial_{t} u+u \partial_{x} u+v \partial_{y} u & =-\partial_{x} p \\
\partial_{x} u+\partial_{y} v & =0
\end{aligned}
$$

with given initial data

$$
u(0, x, y)=u_{0}(x, y)
$$

and boundary condition

$$
v(t, x, 0)=v(t, x, 1)=0,
$$

where $(u, v, p)=(u(t, x, y), v(t, x, y), p(t, x))$ are unknowns.

This system, which describes the leading order behavior of an ideal flow moving in a very narrow domain $\mathbb{R} \times[0, \epsilon]$, can be derived formally by the least action principle Bre08] or a rescaled limit [Lio96](\$4.6). The rescaled limit, called the hydrostatic limit in the literature, can be rigorously justified for the periodic flows under the local Rayleigh condition Bre03, Gre99, MW12].

At the present time, there are no global existence results for (1.1)-(1.4). Even for the local well-posedness, there are only three results. The first result was obtained under the local Rayleigh condition by Y. Brenier in [Bre99, in which the local existence of a special class of classical solutions was proved by applying a semiLagrangian reformulation. Another approach is using the energy method. The authors in MW12 recently established the local well-posedness of $x$-periodic $H^{s}$ solutions under the local Rayleigh condition. Without this condition, the local existence and uniqueness of analytic solutions were also proved by a Cauchy-Kowalevski type argument in KTVZ11.

Received by the editors November 1, 2012 and, in revised form, April 27, 2013.

2010 Mathematics Subject Classification. Primary 35Q31; Secondary 35A01, 35L04, 35L60, 35Q35, 76B99.

Key words and phrases. Formation of singularity, ill-posedness, hydrostatic approximation, classical invariant transformations.

${ }^{1}$ In the literature, it is also called homogeneous hydrostatic equations (see Bre99 for example) or inviscid Prandtl's equations (see E00 for example). 
Regarding the blowup, there is a recent result [CINT] which proved that for a certain class of initial data, spatially symmetric smooth solutions will blow up in finite time.

Our blowup result, which does not rely on any symmetry, is an analogous result for the unsteady Prandtl equations by W. E and B. Engquist EE97. We prove that a smooth solution with a certain class of initial data will blow up in finite time; see Theorem 2.1 for the details. The blowup is either in $\max \left\{\|u(t)\|_{L^{\infty}},\left\|\partial_{x} p(t)\right\|_{L^{\infty}}\right\}$ or $\left\|\partial_{x} u(t)\right\|_{L^{\infty}}$. The first case corresponds to the infinite horizontal velocity or pressure gradient, which is non-physical in a certain sense. The second case corresponds to the formation of singularity.

The main novelty of this proof is to "freeze" a smooth solution by a classical invariant transformation, which will be given in section 3. After "freezing" the solution, we will apply an a priori estimate on the second derivative of the pressure term, which is a simple consequence of Lemma 2.5 below, to derive a Ricatti type inequality. This provides the blowup.

\section{MAIN THEOREM AND ITS PROOF}

The prime objective of this paper is to prove the following:

Theorem 2.1 (Blowup). Let $(u, v, p)$ be a smooth solution to (1.1)-(1.4). Suppose there exist a position $\hat{x} \in \mathbb{R}$ and a constant horizontal velocity $\hat{u} \in \mathbb{R}$ such that the initial data $u_{0}$ satisfies the following properties at $x=\hat{x}$ :

$$
\begin{aligned}
u_{0}(\hat{x}, y) & \equiv \hat{u}, \text { for all } y \in[0,1], \\
\partial_{x y} u_{0}(\hat{x}, 0) & =0, \text { and } \\
\partial_{x y y} u_{0}(\hat{x}, y) & <0, \text { for all } y \in(0,1) .
\end{aligned}
$$

Then there exists a finite time $T>0$ such that if $(u, v, p)$ remains smooth in the time interval $[0, T)$, then either

$$
\lim _{t \rightarrow T^{-}} \max \left\{\|u(t)\|_{L^{\infty}},\left\|\partial_{x} p(t)\right\|_{L^{\infty}}\right\}=+\infty
$$

or

$$
\lim _{t \rightarrow T^{-}} \partial_{x} u(t, X(t, \hat{x}, 1), 1)=-\infty,
$$

where $X(t, \hat{x}, 1)$ is the $x$-component of the characteristid starting from $(\hat{x}, 1)$.

Proof. Our strategy is to show that $\partial_{x} u$ blows up in finite time assuming that both $u$ and $\partial_{x} p$ stay finite. That is, we will prove (2.5) provided that

$$
\max _{t \geq 0}\left\{\|u(t)\|_{L^{\infty}},\left\|\partial_{x} p(t)\right\|_{L^{\infty}}\right\}<+\infty .
$$

Step 1 (Simplify the problem). The first step is to reduce our problem by using the following "freezing" lemma.

Lemma 2.2 (Freezing the solution). Let $(u, v, p)$ be a smooth solution to (1.1)-(1.4) with the initial property (2.1). Assume that there exists a constant $M$ such that

$$
\max _{t \geq 0}\left\{\|u(t)\|_{L^{\infty}},\left\|\partial_{x} p(t)\right\|_{L^{\infty}}\right\} \leq M<+\infty .
$$

\footnotetext{
${ }^{2}$ For the precise definition of $X$, see Definition 3.1
} 
Then there exists a classical invariant transformation

$$
(u, v, p, t, x, y) \mapsto(\tilde{u}, \tilde{v}, \tilde{p}, \tilde{t}, \tilde{x}, \tilde{y})
$$

such that

(i) $(\tilde{u}, \tilde{v}, \tilde{p})$ satisfies (1.1), (1.2) and (1.4),

(ii) $\tilde{u}(\tilde{t}, 0, \tilde{y}) \equiv 0$,

(iii) $\partial_{\tilde{x}} \tilde{u}(\tilde{t}, \tilde{x}, \tilde{y})=\partial_{x} u(t, x, y)$,

(iv) $\partial_{\tilde{x}} \tilde{u}(\tilde{t}, 0,1)=\partial_{x} u(t, X(t, \hat{x}, 1), 1)$,

where $X(t, \hat{x}, 1)$ is the $x$-component of the characteristic of the smooth solution $(u, v, p)$ starting from $(\hat{x}, 1)$.

In addition, if the initial data $u_{0}$ satisfies properties (2.2)-(2.3), then we also have

(v) $\partial_{\tilde{x} \tilde{y}} \tilde{u}(0,0,0)=0$,

(vi) $\partial_{\tilde{x} \tilde{y} \tilde{y}} \tilde{u}(0,0, \tilde{y})<0$ for all $\tilde{y} \in(0,1)$.

Remark 2.3. The classical invariant transformation stated in Lemma 2.2 can be written explicitly as (3.1) below with $g(t):=X\left(t, \hat{x}, y_{0}\right)$ for any $y_{0} \in[0,1]$. Indeed, this $g(t)$ is independent of the choice of $y_{0}$. See section 3 for the details.

The proof of Lemma 2.2 will be given in section 3 . The importance of Lemma 2.2 is that, without loss of generality, we may assume that $\hat{x}=0$ and

$$
u(t, 0, y) \equiv 0, \quad \text { for all }(t, y) \in \mathbb{R}^{+} \times[0,1] .
$$

A direct consequence of (2.6) is

$$
\partial_{y} u(t, 0, y) \equiv 0 .
$$

Furthermore, by Lemma 2.2 again, we can also rewrite our aim (2.5) as

$$
\lim _{t \rightarrow T^{-}} \partial_{x} u(t, 0,1)=-\infty .
$$

Step 2 (Simplify the system). In this step, we will further simplify the system as follows.

Differentiating (1.1) with respect to $x$, we obtain

$$
\partial_{t x} u+u \partial_{x x} u+\left(\partial_{x} u\right)^{2}+v \partial_{x y} u+\partial_{x} v \partial_{y} u=-\partial_{x x} p .
$$

Integrating (2.9) with respect to $y$ over $[0,1]$, using (1.2), (1.4) and the fact that $p$ is independent of $y$, we obtain an integral representation

$$
-\partial_{x x} p=2 \int_{0}^{1} u \partial_{x x} u+\left(\partial_{x} u\right)^{2} d y .
$$

Let $a(t, y):=-\partial_{x} u(t, 0, y), a_{0}(y):=-\partial_{x} u_{0}(0, y)$ and $\mathrm{v}(t, y):=v(t, 0, y)$. Then restricting (2.9) to $x=0$, and using (2.6), (2.7) and (2.10), we have

$$
\partial_{t} a+\mathrm{v}_{y} a=a^{2}-2 \int_{0}^{1} a^{2} d y
$$

It follows from the definition of $a$ and the incompressibility condition (1.2) that

$$
\partial_{y} \mathrm{v}=a,
$$

and hence the boundary condition (1.4) implies that

$$
\mathrm{v}(t, 0)=\mathrm{v}(t, 1)=0 \text { and } \int_{0}^{1} a d y=0 .
$$


Furthermore, the initial data (1.3) gives

$$
a(0, y)=a_{0}(y)
$$

and hypotheses (2.2) and (2.3) become

$$
\begin{gathered}
\partial_{y} a_{0}(0)=0, \\
\partial_{y y} a_{0}>0 .
\end{gathered}
$$

Lastly, our aim (2.8) becomes

$$
\lim _{t \rightarrow T^{-}} a(t, 1)=+\infty .
$$

Step 3 (Blowup estimate). In this step we need two lemmas as follows.

Lemma 2.4. Let $a$ be a smooth solution to (2.11)-(2.14). If $a_{0}$ satisfies (2.15) (2.16), then

$$
\partial_{y} a(t, 0) \equiv 0 \text { and } \partial_{y y} a>0 .
$$

Proof. This proof is based on the method of characteristics. First, differentiating (2.11) with respect to $y$ once, we have

$$
\partial_{t y} a+\mathrm{v} \partial_{y y} a=a \partial_{y} a,
$$

which implies $\partial_{y} a(t, 0) \equiv 0$ provided that $\partial_{y} a_{0}(0)=0$ and $\mathrm{v}(t, 0) \equiv 0$. Second, differentiating (2.11) with respect to $y$ twice, we obtain

$$
\partial_{t y y} a+v_{y y y} a=\left(\partial_{y} a\right)^{2} \geq 0 .
$$

Thus, $\partial_{y y} a$ is increasing along every characteristic, and (2.16) implies $\partial_{y y} a>0$.

Lemma 2.5. Let $f:[0,1] \rightarrow \mathbb{R}$ be a $C^{2}$ function with the following properties:

(i) $f^{\prime}(0)=0$ and $f^{\prime \prime}>0$,

(ii) $\int_{0}^{1} f d y=0$.

Then $f(1)>0$ and

$$
\int_{0}^{1} f^{2} d y \leq \frac{1}{3} f(1)^{2} .
$$

Lemma 2.5] is just an elementary property for convex functions, so we leave it for the reader. For a proof of Lemma 2.5. we refer the reader to Lemma 3.4.3 of Won10 for instance.

Applying these lemmas, we can prove the blowup (2.17) as follows.

It follows from Lemma 2.4 and (2.13) that $a(t, \cdot)$ satisfies the hypotheses of Lemma 2.5, so the $L^{2}$ estimate (2.18) implies that

$$
\partial_{t} a+\mathrm{v} \partial_{y} a=a^{2}-2 \int_{0}^{1} a^{2} d y \geq a^{2}-\frac{2}{3} a(t, 1)^{2} .
$$

Since $\mathrm{v}(t, 1)=0$, we obtain a Ricatti type inequality at $y=1$ :

$$
\partial_{t} a(t, 1) \geq \frac{1}{3} a(t, 1)^{2}
$$

and hence, it follows from the comparison principle of ordinary differential equations that

$$
a(t, 1) \geq \frac{3 a_{0}(1)}{3-a_{0}(1) t}
$$


Finally, applying Lemma 2.5 to $a_{0}$, we have $a_{0}(1)>0$. Since $a_{0}(1)>0$, we know via (2.19) that there exists a finite time $T>0$ such that the blowup (2.17) holds. This completes the proof of Theorem 2.1

\section{BASIC PROPERTIES AND CLASSICAL INVARIANT TRANSFORMATIONS}

The main purpose of this section is to prove Lemma 2.2. To do this, we will first study the basic properties of smooth solutions to (1.1)-(1.4) as follows.

In general, smooth solutions to (1.1)-(1.4) are not unique because the classical invariant transformation group

$$
\begin{cases}\tilde{u}:=u-g^{\prime}, & \tilde{t}:=t, \\ \tilde{v}:=v, & \tilde{x}:=x-g, \\ \tilde{p}:=p+x g^{\prime \prime}, & \tilde{y}:=y\end{cases}
$$

will produce a new solution of (1.1)-(1.4) if $g(0)=g^{\prime}(0)=0$. Here, we can also use this transformation group to prove Lemma 2.2 if we choose $g$ appropriately.

In order to choose a suitable $g$, we study the characteristics given by the following:

Definition 3.1 (Characteristics). The functions $X\left(t, x_{0}, y_{0}\right)$ and $Y\left(t, x_{0}, y_{0}\right)$ are called the $x$-component and $y$-component of the characteristic starting from $\left(x_{0}, y_{0}\right)$ respectively if they satisfy

$$
\left\{\begin{aligned}
\dot{X}\left(t, x_{0}, y_{0}\right) & =u\left(t, X\left(t, x_{0}, y_{0}\right), Y\left(t, x_{0}, y_{0}\right)\right), \\
X\left(0, x_{0}, y_{0}\right) & =x_{0} \\
\dot{Y}\left(t, x_{0}, y_{0}\right) & =v\left(t, X\left(t, x_{0}, y_{0}\right), Y\left(t, x_{0}, y_{0}\right)\right), \\
Y\left(0, x_{0}, y_{0}\right) & =y_{0}
\end{aligned}\right.
$$

where the dot represents $\frac{d}{d t}$. We may also write $X(t)$ and $Y(t)$ if there is no ambiguity.

The characteristic $X(t)$ of a smooth solution $(u, v, p)$ of the hydrostatic Euler equations (1.1)-(1.2) has an interesting property, which can be stated as follows:

Proposition 3.2. If $u_{0}\left(x_{0}, y_{0}\right)=u_{0}\left(x_{0}, y_{1}\right)$, then for any $t \geq 0$,

$$
\begin{aligned}
X\left(t, x_{0}, y_{0}\right) & =X\left(t, x_{0}, y_{1}\right), \text { and } \\
u\left(t, X\left(t, x_{0}, y_{0}\right), Y\left(t, x_{0}, y_{0}\right)\right) & =u\left(t, X\left(t, x_{0}, y_{1}\right), Y\left(t, x_{0}, y_{1}\right)\right) .
\end{aligned}
$$

Proof. The equality (3.2) follows directly from the uniqueness of ordinary differential equations because both $X\left(t, x_{0}, y_{0}\right)$ and $X\left(t, x_{0}, y_{1}\right)$ satisfy the same ordinary differential equation

$$
\ddot{X}=-\partial_{x} p(t, X)
$$

with the same initial data

$$
\left\{\begin{array}{l}
X(0)=x_{0} \\
\dot{X}(0)=u_{0}\left(x_{0}, y_{0}\right) .
\end{array}\right.
$$

Equality (3.3) can be obtained by differentiating (3.2) with respect to $t$.

Proposition 3.2 and the boundary condition (1.4) give us a useful

Corollary 3.3. $X\left(t, \hat{x}, y_{0}\right)$ and $u\left(t, X\left(t, \hat{x}, y_{0}\right), y\right)$ are also independent of $y_{0}$ and $y$ if $u_{0}\left(\hat{x}, y_{0}\right)$ is independent of $y_{0}$. 
Now, using the knowledge above, we are going to prove Lemma 2.2 as follows.

From the hypothesis (2.1), we know that $u_{0}\left(\hat{x}, y_{0}\right)$ is independent of $y_{0}$, so by Corollary 3.3. $X\left(t, \hat{x}, y_{0}\right)$ is independent of $y_{0}$. Therefore, we can choose $g(t):=$ $X\left(t, \hat{x}, y_{0}\right)$ for any $y_{0} \in[0,1]$. Since $\left|g^{\prime}(t)\right|=|\dot{X}|=|u(t, X, Y)| \leq M$ and $\left|g^{\prime \prime}(t)\right|=$ $|\ddot{X}|=\left|\partial_{x} p(t, X)\right| \leq M$, we can apply the invariant transformation (3.1) as long as $(u, v, p)$ is smooth.

To complete the proof, we have to check that this transformation satisfies parts (i)-(vi) in Lemma 2.2, By direct computations, parts (i), (iii), (v) and (vi) can be checked easily by using the following two facts:

(a) $\partial_{\tilde{t}}=\partial_{t}+g^{\prime} \partial_{x}, \partial_{\tilde{x}}=\partial_{x}$ and $\partial_{\tilde{y}}=\partial_{y}$,

(b) $g(0)=\hat{x}$.

Furthermore, part (ii) holds because

$$
\begin{aligned}
\tilde{u}(\tilde{t}, 0, \tilde{y}) & =u(\tilde{t}, g(\tilde{t}), \tilde{y})-g^{\prime}(\tilde{t}) \\
& =u\left(\tilde{t}, X\left(\tilde{t}, \hat{x}, y_{0}\right), \tilde{y}\right)-u\left(\tilde{t}, X\left(\tilde{t}, \hat{x}, y_{0}\right), Y\left(\tilde{t}, \hat{x}, y_{0}\right)\right) \\
& =0,
\end{aligned}
$$

where we applied Corollary 3.3 in the last equality.

Lastly, part (iv) follows directly from part (iii): since $g(t)=X(t, \hat{x}, 1)$,

$$
\partial_{\tilde{x}} \tilde{u}(\tilde{t}, 0,1)=\partial_{x} u(t, g(t), 1)=\partial_{x} u(t, X(t, \hat{x}, 1), 1) .
$$

This completes the proof of Lemma 2.2

Remark 3.4. One may conclude this section with a thought experiment: Imagine that there is a water flow $(u, v, p)$ in an infinitely long river $\mathbb{R} \times[0,1]$. As a stationary observer, you can stand at a fixed point on the river bank, say the origin; then you will see the flow as $(u, v, p)$. On the other hand, you can move parallel to the river bank with velocity $g^{\prime}(t)$, then you will see the flow as $(\tilde{u}, \tilde{v}, \tilde{p})$. In other words, both $(u, v, p)$ and $(\tilde{u}, \tilde{v}, \tilde{p})$ describe the same physical phenomenon but with different reference frames, so Lemma 2.2 is just stating that if the initial horizontal velocity $u_{0}$ has a line $x=\hat{x}$ with a constant horizontal velocity $\hat{u}$, then you can freeze this constant horizontal velocity line provided that you move appropriately.

Another physical interpretation that can be made from this thought experiment is that the blowup (2.4) of $u$ or $\partial_{x} p$ can be seen as the consequence of an observer moving at an infinite speed or accelerating at an infinite rate respectively.

\section{REFERENCES}

[Bre99] Yann Brenier, Homogeneous hydrostatic flows with convex velocity profiles, Nonlinearity 12 (1999), no. 3, 495-512, DOI 10.1088/0951-7715/12/3/004. MR:1690189 (2000b:35205)

[Bre03] Yann Brenier, Remarks on the derivation of the hydrostatic Euler equations (English, with English and French summaries), Bull. Sci. Math. 127 (2003), no. 7, 585-595, DOI 10.1016/S0007-4497(03)00024-1. MR2004720 (2004g:76013)

[Bre08] Yann Brenier, Generalized solutions and hydrostatic approximation of the Euler equations, Phys. D 237 (2008), no. 14-17, 1982-1988, DOI 10.1016/j.physd.2008.02.026. MR2449787 (2010a:76074)

[CINT] Chongsheng Cao, Slim Ibrahim, Kenji Nakanishi, and Edriss S. Titi, Finite-time blowup for the inviscid primitive equations of oceanic and atomspheric dynamics, arXiv:1210.7337v1 [math.AP].

[E00] Weinan E, Boundary layer theory and the zero-viscosity limit of the NavierStokes equation, Acta Math. Sin. (Engl. Ser.) 16 (2000), no. 2, 207-218, DOI 10.1007/s101140000034. MR.1778702(2001d:76037) 
[EE97] Weinan E and Bjorn Engquist, Blowup of solutions of the unsteady Prandtl's equation, Comm. Pure Appl. Math. 50 (1997), no. 12, 1287-1293, DOI 10.1002/(SICI)10970312(199712)50:12〈1287::AID-CPA4〉3.0.CO;2-4. MR1476316 (99c:35196)

[Gre99] Emmanuel Grenier, On the derivation of homogeneous hydrostatic equations, M2AN Math. Model. Numer. Anal. 33 (1999), no. 5, 965-970, DOI 10.1051/m2an:1999128. MR.1726718 (2000j:76051)

[KTVZ11] Igor Kukavica, Roger Temam, Vlad C. Vicol, and Mohammed Ziane, Local existence and uniqueness for the hydrostatic Euler equations on a bounded domain, J. Differential Equations 250 (2011), no. 3, 1719-1746, DOI 10.1016/j.jde.2010.07.032. MR2737223 (2011j:76010)

[Lio96] Pierre-Louis Lions, Mathematical topics in fluid mechanics. Vol. 1, Oxford Lecture Series in Mathematics and its Applications, vol. 3, The Clarendon Press, Oxford University Press, New York, 1996. Incompressible models; Oxford Science Publications. MR:1422251 (98b:76001)

[MW12] Nader Masmoudi and Tak Kwong Wong, On the $H^{s}$ theory of hydrostatic Euler equations, Arch. Ration. Mech. Anal. 204 (2012), no. 1, 231-271, DOI 10.1007/s00205-0110485-0. MR2898740

[Won10] Tak Kwong Wong, On the wellposedness of boundary layer equations, Ph.D. thesis, New York University, 2010.

Department of Mathematics, University of Pennsylvania, David Rittenhouse LaboRatory, 209 South 33rd Street, Philadelphia, Pennsylvania 19104-6395

E-mail address: takwong@math.upenn.edu 